ARTICLE

Received 11 Sep 2015 | Accepted 2 Mar 2016 | Published 4 Apr $2016 \quad$ DOl: 10.1038/ncomms11211

\title{
Prediction of a native ferroelectric metal
}

\author{
Alessio Filippetti ${ }^{1}$, Vincenzo Fiorentini ${ }^{1,2}$, Francesco Ricci ${ }^{2,3}$, Pietro Delugas ${ }^{4,5}$ \& Jorge Íñiguez ${ }^{6,7}$
}

Over 50 years ago, Anderson and Blount discussed symmetry-allowed polar distortions in metals, spawning the idea that a material might be simultaneously metallic and ferroelectric. While many studies have ever since considered such or similar situations, actual ferroelectricity - that is, the existence of a switchable intrinsic electric polarization-has not yet been attained in a metal, and is in fact generally deemed incompatible with the screening by mobile conduction charges. Here we refute this common wisdom and show, by means of first-principles simulations, that native metallicity and ferroelectricity coexist in the layered perovskite $\mathrm{Bi}_{5} \mathrm{Ti}_{5} \mathrm{O}_{17}$. We show that, despite being a metal, $\mathrm{Bi}_{5} \mathrm{Ti}_{5} \mathrm{O}_{17}$ can sustain a sizable potential drop along the polar direction, as needed to reverse its polarization by an external bias. We also reveal striking behaviours, as the self-screening mechanism at work in thin $\mathrm{Bi}_{5} \mathrm{Ti}_{5} \mathrm{O}_{17}$ layers, emerging from the interplay between polar distortions and carriers in this compound.

\footnotetext{
${ }^{1}$ CNR-IOM SLACS Cagliari, Istituto Officina dei Materiali, Cittadella Universitaria, Monserrato (CA) 09042-I, Italy. ${ }^{2}$ Dipartimento di Fisica, Universita' di Cagliari, Cittadella Universitaria, Monserrato (CA) 09042-I, Italy. ${ }^{3}$ Institute of Condensed Matter and Nanosciences, Université Catholique de Louvain, 1348 Louvain-la-Neuve, Belgium. ${ }^{4}$ Istituto Italiano di Tecnologia, Via Morego 30, 16163 Genova, Italy. ${ }^{5}$ Scuola Internazionale Superiore di Studi Avanzati, Via Bonomea 265, 34136 Trieste, Italy. ${ }^{6}$ Department of Materials Research and Technology, Luxembourg Institute of Science and Technology, 5 Avenue des Hauts-Fourneaux, L-4362 Esch/Alzette, Luxembourg. ${ }^{7}$ Institut de Ciència de Materials de Barcelona (ICMAB-CSIC), Campus UAB, 08193 Bellaterra, Spain. Correspondence and requests for materials should be addressed to A.F. (email: alessio.filippetti@dsf.unica.it) or to V.F. (email: vincenzo.fiorentini@dsf.unica.it) or to J.í. (email: jorge.iniguez@list.lu).
} 
$\mathrm{T}$ he possibility that metals may exhibit ferroelectricity is an intriguing open issue. Anderson and Blount ${ }^{1}$ showed that certain martensitic transitions involve inversion symmetry breaking and, formally, the existence of a polar axis. Metallic ferroelectric behaviour has thus been claimed for metals undergoing a centrosymmetric (CS) to non-CS structural transformation, such as ${ }^{2,3} \mathrm{Cd}_{2} \mathrm{ReO}_{7}$ and $\mathrm{LiOsO}_{3}$, or being natively non-CS, such as ${ }^{4}(\mathrm{Sr}, \mathrm{Ca}) \mathrm{Ru}_{2} \mathrm{O}_{6}$. The same label has been attached to ferroelectric insulators whose polar distortion survives moderate metallicity induced by doping or proximity ${ }^{5-7}$. However, it seems fair to say that none of these systems, nor any other to our knowledge, embodies a truly ferroelectric metal with native switchable polarization and native metallicity coexisting in a single phase.

Here we report a theoretical prediction of such a material. By first-principles calculations, we show that the layered perovskite $\mathrm{Bi}_{5} \mathrm{Ti}_{5} \mathrm{O}_{17}$ has a non-zero density of states (DOSs) at the Fermi level and metal-like conductivity, as well as a spontaneous polarization in zero field. Further, we predict that the polarization of $\mathrm{Bi}_{5} \mathrm{Ti}_{5} \mathrm{O}_{17}$ is switchable both in principle (the material complies with the sufficient symmetry requirements) and in practice (in spite of being a metal, $\mathrm{Bi}_{5} \mathrm{Ti}_{5} \mathrm{O}_{17}$ can sustain a sizable potential drop along the polar direction, as needed to revert its polarization by application of an electric bias). Beyond their conceptual importance, our results reveal striking behaviours-such as a selfscreening mechanism at work in thin $\mathrm{Bi}_{5} \mathrm{Ti}_{5} \mathrm{O}_{17}$ layers-emerging from the intimate interplay between polar distortions and free carriers. Our results thus challenge the common wisdom regarding the possibilities to control charges and fields at the nano-scale, with exciting potential implications in areas ranging from photovoltaics to electronics.

\section{Results}

Non-CS metallic ground state in $\mathbf{B i}_{5} \mathbf{T i}_{5} \mathbf{O}_{17}$. We focus on layered perovskite titanates $\mathrm{A}_{n} \mathrm{Ti}_{n} \mathrm{O}_{3 n+2}$ (refs 8,9), whose structure foreshadowing low-dimensional behaviour combines with their tunable conduction charge: assuming fixed ionic charges for $\mathrm{A}^{3+}$ and $\mathrm{O}^{2-}$, Ti has nominal oxidation state of $(3+4 / n)$, that is, between $4+$ for $n=4$ (for example, the band insulator, high-temperature ferroelectric $\left.\mathrm{La}_{2} \mathrm{Ti}_{2} \mathrm{O}_{7}\right)$ and $3+$ in the $n \rightarrow \infty$ limit (for example, the Mott-insulating Ti- $3 \mathrm{~d}^{1}$ perovskite $\mathrm{LaTiO}_{3}$ ). The metallic $n>4$ phases are not nearly as studied as the end compounds ${ }^{10-12}$. Motivated by experimental reports of possible non-CS structures in the $n=5$ compound $\mathrm{La}_{5} \mathrm{Ti}_{5} \mathrm{O}_{17}$ (La-5517 hereafter) ${ }^{13}$, here we discuss this material as well as the alternative composition $\mathrm{Bi}_{5} \mathrm{Ti}_{5} \mathrm{O}_{17}$ (Bi-5517).

The $n=5$ layered titanate can be viewed as a stack of slabs containing 5 [011]-oriented perovskite-like planes and AO terminated. (See Fig. 1, as well as Supplementary Fig. 1 and Supplementary Note 1, for details. Directions are given in the pseudo-cubic setting of the perovskite structure.) The crystal axes are $\mathbf{b}=[011]$, which will be shown to coincide with the polar axis, and $\mathbf{a}=[100]$ and $\mathbf{c}=[0-11]$, which define the plane where the conduction charge is largely confined. Our simulation supercell comprises two five-layer blocks along $\mathbf{b}$ and is compatible with all structures of interest here. To identify the ground state, we start from the high-symmetry structure (Immm space group, Fig. 1a) and condense all its unstable distortions as obtained from a Hessian analysis. (See the Methods for details of the firstprinciples simulations.) For La-5517, the ground state is CS Pmnn (Fig. 1b), barring the existence of polarization. We also simulate the experimentally proposed structure of ref. 13 , but find it to be a high-energy unstable configuration.

Inspired by the observation that perovskites where $\mathrm{Bi}^{3+}$ replaces $\mathrm{La}^{3+}$ tend to be ferroelectric due to $\mathrm{Bi}^{3+}$ 's tendency to form low-coordination complexes with neighbouring a

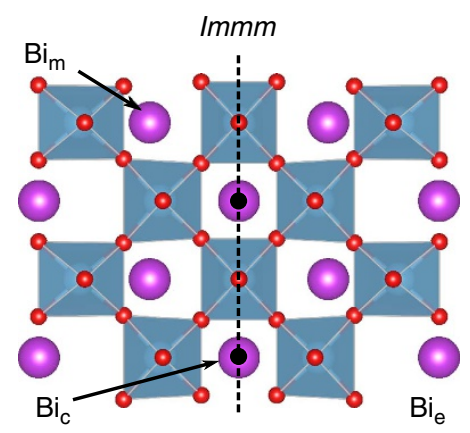

b
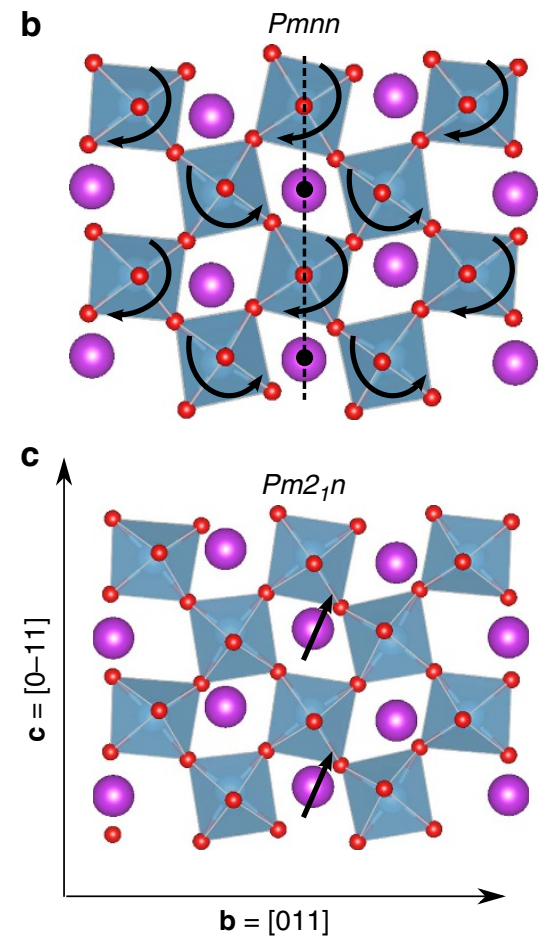

Figure $\mathbf{1}$ | Structural detail of $\mathbf{B i}_{\mathbf{5}} \mathbf{T i}_{\mathbf{5}} \mathbf{O}_{\mathbf{1 7}}$ phases. Sketch of the $\mathrm{Bi}-5517$ phases discussed in the text, showing relevant structural features. Large violet balls stand for $\mathrm{Bi}$ atoms, small red balls for oxygens, green polyhedra for the $\mathrm{TiO}_{6}$ groups. Most relevant atomic distortions indicated with arrows. For each structure, black lines outline the supercell profile (a) $I \mathrm{mmm}$,

(b) Pmnn, (c) Pm2 ${ }_{n}$. Only one b-oriented slab is shown (there are two such slabs in the simulation cell). Bi types indicated in panel (a). b-oriented mirror plane, and inversion centres at the $\mathrm{Bi}_{\mathrm{c}}$ positions, indicated in panels $(\mathbf{a}, \mathbf{b})$. In the Pmnn case, this mirror plane has an associated $1 / 2(\mathbf{a}+\mathbf{b}+\mathbf{c})$ glide translation; hence, the symmetry is not obvious from the figure. $\mathrm{Bi}_{\mathrm{c}}$ off-centering displacements indicated in panel $(\mathbf{c})$. The $\mathrm{Bi}_{\mathrm{c}}$ displacement along $\mathbf{c}$ is compensated by with a symmetric one occurring in the second slab in the cell (not shown); the $\mathrm{Bi}_{\mathrm{c}}$ displacement along $\mathbf{b}$ adds up with the symmetric one in the second slab, and thus a b-oriented polarization appears.

oxygens $^{14,15}$, we explore symmetry breaking in Bi-5517, obtained by replacing all La atoms with Bi's. As in La-5517, the Immm phase is a high-energy saddle point. However, at variance with La-5517, the Pmnn structure is also a saddle point for Bi-5517. We then condense the unstable distortions of this phase, and identify as lowest-energy solution a structure with the non-CS $\mathrm{Pm}_{1} n$ space group (Fig. 1c). We computed the Hessian for this $P m 2_{1} n$ structure and confirmed it to be a minimum of the energy. The symmetry breaking distortion in the $\mathrm{Pm}_{1} n$ phase can be appreciated by looking at the Bi's in the central layer $\left(B i_{c}\right.$ in Fig. 1): although they remain at high-symmetry CS 
positions in both Immm and Pmnn, they move off-centre in $P m 2_{1} n$, thus breaking the (011) mirror plane and yielding a symmetrywise ferroelectric structure.

As regards the electronic structure, Bi-5517's $P m 2_{1} n$ phase is clearly metallic, as can be seen in Fig. 2a-e from the atom- and orbital-resolved DOS. We have two conduction electrons per primitive cell (that is, a density of $3 \times 10^{21} \mathrm{~cm}^{-3}$ ), with $E_{\mathrm{F}}$ crossing the $\mathrm{Ti} 3 d-t_{2 \mathrm{~g}}$ band manifold $\sim 0.4 \mathrm{eV}$ above the conduction band bottom (CBB). The near-CBB DOS highlights a marked two-dimensional character, with $40 \%$ of the conduction charge confined within the central Ti layer of each block, $25 \%$ in each of the two intermediate layers and only $5 \%$ in each of the edge Ti's. The $t_{2 \mathrm{~g}} \mathrm{CBB}$ is split into $d_{y z}$ (laying orthogonally to the stacking plane, rising in energy due to reduced hopping along the stacking direction) and $d_{x y} / d_{x z}$ states (the hopping along $x$ being unaffected by the stacking). $d_{x y}$ and $d_{x z}$ are also split: only $d_{x y}$ has significant DOS below $E_{\mathrm{F}}$ in one of the five-layer blocks, and only $d_{x z}$ in the other, signalling orbital ordering. Figure $2 \mathrm{f}$ highlights the anisotropy of the conduction bands: the two occupied bands per block are doubled, there being two blocks in the supercell; yet, the splitting due to inter-block coupling is negligible, confirming good confinement of the conduction electrons within each block. The inset of Fig. $2 \mathrm{f}$ shows that the bands are completely flat along the $\Gamma$-Y (stacking) direction, with no band crossing $E_{\mathrm{F}}$. The system is thus gapped at $\Gamma$ along this direction, although of course it may not be for a generic $k$-point away from zone centre.

Figure $2 \mathrm{~g}$ shows the Fermi surface (FS). The lowest-energy band $S_{1}$ consists of two disconnected parallel sheets, and the higher $\mathrm{S}_{2}$ band contributes an elliptic tube. Along $\Gamma-Y$ (b direction), the FS is very flat and resistivity is high, as shown in Fig. 2h. Along $\Gamma$-X (a direction), the light-mass $S_{2}$ contributes to mobility, while $S_{1}$ is disconnected. Finally, along $\Gamma-Z$ (c direction), both sections contribute, but yield relatively high resistivity as the corresponding masses are much heavier than along $\Gamma$-X. As a result, the predominant low-resistivity channel is largely one-dimensional along a; nevertheless, the resistivity temperature dependence is that of a metal in all directions. The ordering of the resistivities is quite consistent with experiments 9 for La-5517, and the weak insulating upturn observed in La-5517 can be reproduced by inserting small defect-like activation energies in the conductivity model (see Supplementary Fig. 2 and Supplementary Note 2 for details).

One might wonder whether the metallic $P m 2_{1} n$ structure could experience additional symmetry-breaking distortions (for example, of the Peierls or Jahn-Teller type) that might open a gap within the conduction band and render an insulating solution. If they existed, such gap-opening distortions would appear as (soft-mode) instabilities of the $P m 2_{1} n$ phase, that is, they would have negative eigenvalues of the corresponding Hessian matrix associated to them. As mentioned above, we explicitly checked that no such soft mode exists, and that the $P m 2_{1} n$ phase is a stable energy minimum. Hence, the metallic character of Bi-5517's non-CS ground state is robust.

Electric polarization and self-screening in $\mathbf{B i}_{5} \mathbf{T i}_{5} \mathbf{O}_{17}$. We now tackle the calculation of the ferroelectric polarization appearing in the $P m 2_{1} n$ phase of $\mathrm{Bi}-5517$. Let us first note that the
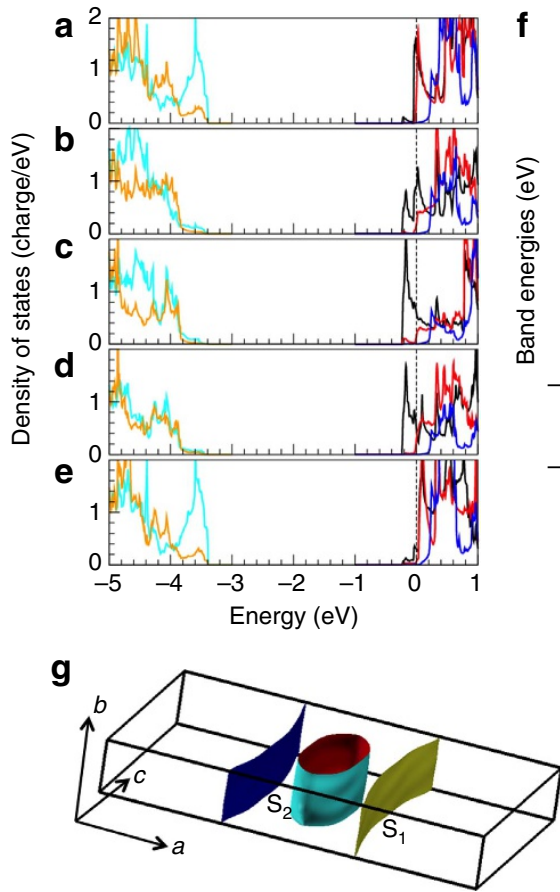
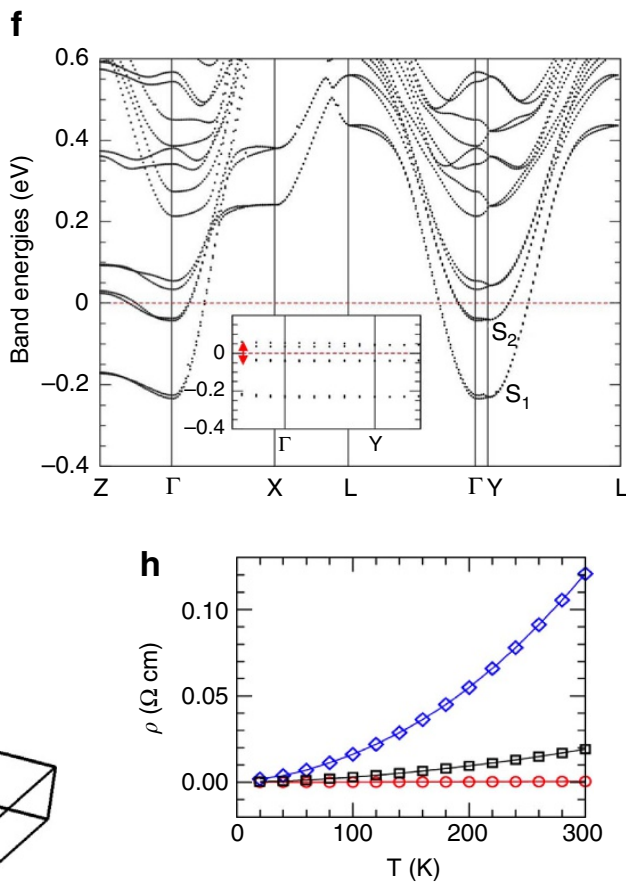

Figure 2 I Main features of the electronic structure of $\mathbf{B i}_{\mathbf{5}} \mathbf{T i}_{\mathbf{5}} \mathbf{O}_{\mathbf{1 7}}$. (a-e) Orbital- and atom-resolved DOS of Pm2 $n$ Bi-5517. The five panels correspond to the five TiO (011) layers in one slab: (c) corresponds to the middle layer, (a,e) to the layers at the stacking, (b,d) to the intermediate layers. For each panel, the DOS is split in individual orbital contributions, indicated with different colours (only the most significant orbital components are displayed): Ti $d_{x y}$ (black lines), Ti $d_{x z}$ (red lines), Ti $d_{y z}$ (blue lines), O $p$ ligand parallel to the (011) planes (orange lines), and O $p$ ligand orthogonal to (011) planes (cyan lines). For the $t_{2 g}$ orbitals, $x, y$ and $z$ correspond to the [100], [010] and [001] directions, respectively. (f) Band structure in the surrounding of the conduction band bottom. Inset: zoom along the $\Gamma$-Y direction. $\mathrm{S}_{1}$ and $\mathrm{S}_{2}$ label the two occupied conduction bands, and the red-arrowed line the gap ( $\Delta E=0.1 \mathrm{eV}$ ) along $\Gamma-Y$ for $k_{\mathrm{x}}=0$. (g) Calculated Fermi surfaces. The bounding box indicates the Brillouin zone used for the calculations, and the Cartesian axes are indicated by the arrows. $S_{1}$ and $S_{2}$ label the Fermi surfaces corresponding to the two occupied conduction bands. $S_{1}$ is composed of two disconnected sheets (in green and violet), which are flat along $b$ and slightly bumped along $c . S_{2}$ is a single flat tube (cyan) of ellipsoidal section parallel to $b$ and rounded along $a$. (h) Resistivity $\rho$ versus temperature calculated along the supercell axes: $\mathbf{a}=[100]$ (red circlets), $\mathbf{b}=[011]$ (blue diamonds), $\mathbf{c}=[0-11]$ (black squares). 
polarization-defined as the integrated current flowing along the stacking direction when we move from the CS phase (Immm) to the non-CS one $(P m 2 n)$ - can be split into contributions from ionic cores, valence electrons and conduction electrons. The first two dominate the effect and are trivial to compute by standard methods ${ }^{16,17}$ : we obtain $P_{\text {ion }}=55.5 \mu \mathrm{Ccm}^{-2}$ and $P_{\text {val }}=-14.6 \mu \mathrm{Ccm}^{-2}$. In contrast, calculating $P_{\text {cond }}$ is not standard. Nevertheless, we can take advantage of the localized character of conduction electrons within the Bi-5517 blocks and implement two independent approaches to calculate $P_{\text {cond }}$. These approaches provide consistent results.

First, we compute $P_{\text {cond }}$ from the dipole associated to conduction electrons within a five-layer block in Bi-5517. Figure 3 shows the planar-averaged conduction charge of the $P m 2{ }_{1} n$ phase, as well as that of a CS system with $P m 2_{1} n$ cell parameters and Immm atomic positions. The $P m 2{ }_{1} n$ phase displays an evident inversion symmetry breaking; a dipole appears within each block and we obtain $P_{\text {cond }}=-4.0 \mu \mathrm{Ccm}^{-2}$. Note that, again, a significant two-dimensional (2D) charge confinement is apparent in Fig. 3, and this strategy to compute $P_{\text {cond }}$ would be exact if the conduction charge were strictly confined within the blocks.

Alternatively, we can compute $P_{\text {cond }}$ using a modified version of the Berry phase formalism. As the occupied conduction bands are rather flat along the $\Gamma$-Y direction of the Brillouin zone (the reciprocal-space signature of confinement), we can generalize the usual formulation to allow for changing numbers of contributing bands at different $k$-point strings (see the Methods for details). We eventually obtain $P_{\text {cond }}=-7.5 \mu \mathrm{Cm}^{-2}$, which we deem in reasonable agreement with our estimate above.

Figure 3 also reveals a fascinating effect, namely, how the mobile carriers rearrange within each of the five-layer blocks to screen the field created by the local dipoles resulting from the CS-breaking displacements of the central $\mathrm{Bi}$ cations (see the respective enhancement and decrease in electron density on the right and left sides of the $\mathrm{Bi}_{\mathrm{c}}$ planes). Remarkably, the ferroelectric instability persists in spite of this self-screening mechanism, contradicting the general notion that an abundance of mobile carriers should prevent any such polar distortion. This result highlights the difference between our material (whose ferroelectric phase has a local, chemical origin associated to the $\mathrm{Bi}-\mathrm{O}$ bonding) and compounds such as $\mathrm{BaTiO}_{3}$ (where the

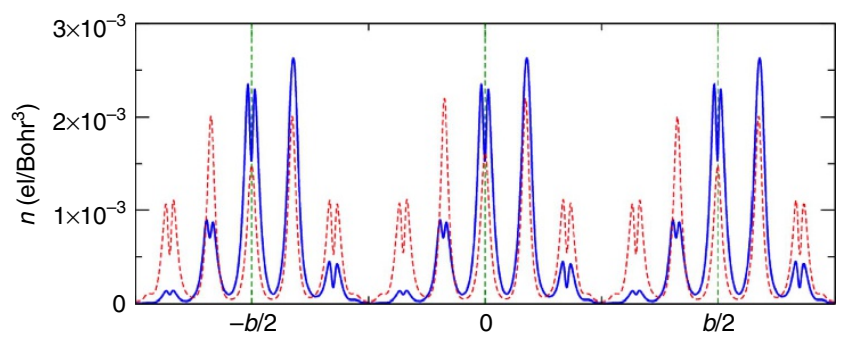

Figure 3 | Conduction charge density of $\mathbf{B i}_{\mathbf{5}} \mathbf{T i}_{\mathbf{5}} \mathbf{O}_{\mathbf{1 7}}$. The density is obtained by means of a planar average of the calculated three-dimensional conduction densities along the stacking direction [011]. Dashed green vertical lines indicate the position of the central Ti layer of a given block; the length $b$ of the supercell along [011] includes two blocks. The blue solid line is the conduction charge density of the electrically polarized $P m 2_{1} n$ structure; the red dashed line is the conduction charge density calculated for a fictitious centrosymmetric system with the same cell parameters of the $P m{ }_{1} n$ structure but internal atomic positions of the centrosymmetric Immm structure. The density is first calculated on the Fast-Fourier Transform grid, and then interpolated on a ultra-dense grid to reduce real-space integration errors. The polarization in the $P m{ }_{1} n$ phase points to the right, whereas it is perfectly vanishing for the centrosymmetric phase. ferroelectric instability relies on the action of dipole-dipole interactions ${ }^{18}$ that are strongly weakened by screening charges ${ }^{5}$ ). Note that the difference in behaviour between chemically driven and dipole-dipole-driven ferroelectrics is well illustrated by the predicted response of prototypical compounds such as $\mathrm{BiFeO}_{3}$ (similar to $\mathrm{Bi}-5517$ in that $\mathrm{Bi}-\mathrm{O}$ bonding dominates the ferroelectric distortion ${ }^{15,19,20}$ ) and $\mathrm{BaTiO}_{3}$ to electron doping and the accompanying metallization: the doping is not detrimental to the polar distortion in the former ${ }^{21}$, but annihilates it in the latter ${ }^{5}$.

Hence, our calculations indicate that the $P m 2_{1} n$ phase of Bi-5517 has a spontaneous polarization of $\sim 35 \mu \mathrm{Cm}^{-2}$, which is in the same league as the most common ferroelectric perovskites (for example, $30 \mu \mathrm{Ccm}^{-2}$ for $\mathrm{BaTiO}_{3}$ ). Ferroelectricity largely originates from $\mathrm{Bi}^{3+}$ cations moving off-centre in the perovskite framework. This displacement is invertible with respect to the (011) plane, so that switching between two equivalent polar states is possible symmetrywise. The computed ferroelectric well depth of $0.31 \mathrm{meV} \AA^{-3}$ suggests a critical temperature upward of $500 \mathrm{~K}$.

\section{Discussion}

So far, we have shown that metallicity coexist with zero-field polarization in $\mathrm{Bi}-5517$. Is it possible to switch the polarization of this ferroelectric metal? Can a finite Bi-5517 sample sustain a finite field, as would be required to switch its polarization? For Bi-5517 in the usual capacitor configuration with metallic electrodes, one may expect the bias to induce a current rather than to act on the polar distortion. For Bi-5517 cladded within insulating layers, current flow is precluded by construction (neglecting tunneling), but mobile carriers should screen an applied bias, and leave the CS-breaking distortion unaffected. It turns out that $\mathrm{Bi}-5517$ is quite at odds with this reasonable expectation. We show this by studying a superlattice (SL) of alternating $\mathrm{Bi}-5517$ (one primitive cell, $\sim 31 \AA$ thick) and $\mathrm{Bi}_{2} \mathrm{Zr}_{2} \mathrm{O}_{7}$ (BZO-227, $n=4$ of the same family; layer $\sim 26 \AA$ thick) layers. BZO-227 acts as a cladding insulator providing seamless stoichiometric continuity on the A-cation site as well as effective confinement of the conduction electrons within $\mathrm{Bi}-5517$ (see Supplementary Fig. 3 and Supplementary Discussion for details). We compare a SL where Bi-5517 is non-CS (starting from the $P m 2_{1} n$ bulk phase) with a reference SL that is a suitable symmetrization of the non-CS one (which yields a Pmnn-like structure for the Bi-5517 layer).

Figure 4 shows planar and filter averages ${ }^{7}$ (see the Methods) of the potentials and conduction densities of the two SLs, and their differences. The key result is the sizable depolarizing field $E_{\text {dep }} \sim 20 \mathrm{MV} \mathrm{m}^{-1}=0.02 \mathrm{GV} \mathrm{m}^{-1}$, which we estimate from the potential slope in the central region of Bi-5517 (see Fig. 4, bottom, for details). Thus, the mobile charge dominates the screening process, but is unable to screen out entirely the polarizationinduced field. (This residual depolarizing field increases further if the number of mobile electrons is reduced by hole injection; see Supplementary Fig. 4 and Supplementary Discussion for details.) The difference between the non-CS and CS conduction densities (Fig. 4, top and centre panel) clearly shows, first, the local self-screening within each five-layer block already observed in the bulk case; and second, a net charge imbalance-with negative and positive carriers accumulating, respectively, at the right (Bi-5517/BZO-227) and left (BZO-227/Bi-5517) interfaces-that acts against the polarization-generated field. Although the overall self-screening response is incomplete, it is still amply sufficient to stabilize the mono-domain polar state even under such unfavourable electrical boundary conditions. (Owing to the $\nabla D=\rho_{\text {free }}=0$ condition across the interface, the mono-domain configuration of thin ferroelectric layers in a ferroelectric- 


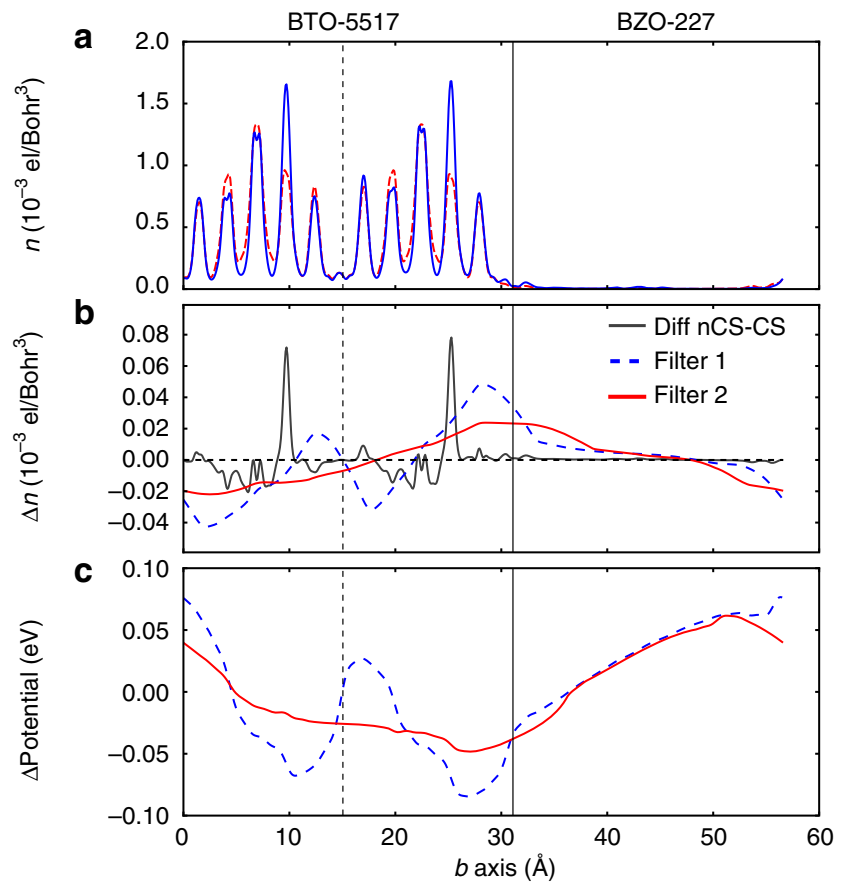

Figure 4 | Potential and conduction charge density of $\mathrm{Bi}_{5} \mathrm{Ti}_{5} \mathrm{O}_{17} / \mathrm{Bi}_{2} \mathrm{Zr}_{2} \mathrm{O}_{7}$ superlattices. Bi-5517 is on the left, BZO-227 on the right, separated by vertical solid lines; dashed vertical lines mark the two Bi-5517 blocks. Horizontal line in $\mathbf{b}$ is the zero density baseline. (a) The planar-averaged conduction density of SL with non-CS (blue solid line) and CS (red dashed) Bi-5517 is shown. (b) Density differences of SL between the non-CS and CS cases in three variants are shown: planar-averaged (grey, thin, solid) reduced by a factor 10 ; filtered with filter $[2 d, 3 d]$ (labelled 2; red, thick, solid); filtered with filter $[2 d, d]$ (labelled 1, blue, thick, dashed). See the Methods for details. (c) Filtered difference of the non-CS and CS potentials (same filters and line convention as panel $\mathbf{b}$ ) is shown. Note that the substantial $(2 \mathrm{eV})$ interface band offset of Bi-5517 to BZO-227 cancels out in the potential difference (c), but leads to efficient confinement (a) of the conduction charge inside Bi-5517. The polarization of the non-CS phase points to the right.

dielectric SL is generally unstable; see Supplementary Discussion for details.) Indeed, explicitly relaxing the non-CS SL, we find that the Bi-5517 layer is almost identical to the polar bulk phase, which confirms the stability of the mono-domain configuration.

The switching of polarization, and more generally the response to an applied field, in Bi-5517 is a non-trivial problem. However, our finite Bi-5517 cladded layer is in an open-circuit configuration where, because of the insulating BZO-227 layers, no current flows; in this case, a plausible argument can be made for the switching. Consider the situation shown in Fig. 4, where the polarization (mostly due to ions) points towards the right side of the layer. The layer screens the polarization-induced field incompletely, so that there is a residual depolarizing field that points from right to left and acts against the polarization. The incomplete screening in itself implies that the layer is effectively a dielectric medium with finite low-frequency, low-wavevector dielectric function. Hence, it is natural to conclude that, upon application of a field exceeding the finite screening ability of the Bi-5517 layer, the polarization will switch. Pictorially, an external field pointing towards the left will push cations (oxygens) to the left (right), whereas valence electrons and mobile conduction electrons go to the right. Yet, the latter response is necessarily limited by the Bi-5517 layer's modest stock of mobile charges and the finiteness of the system, and hence the screening will be incomplete. Note that, in contrast, ferroelectric switching seems less likely in a bulk Bi-5517 sample; in that case, the reservoir of mobile carriers is essentially unlimited, and the details of the dynamical response of electrons and lattice to the external bias will probably become critical to decide whether switching can be achieved or not.

As just shown, even a unit cell of single-domain Bi-5517 is polarized and sustains a polarization-generated field. Bi-5517 thus contradicts the natural assumption that, in the nanometric-film limit ${ }^{6}$, polarization can never survive its own depolarization field. Indeed, Bi-5517 stands apart from any other known ferroelectric material because of the coexistence of a localized and strong polar instability (driven by the formation of $\mathrm{Bi}-\mathrm{O}$ bonds) and a self-screening mechanism that does not prevent the chemically driven polar distortion but does partly cancel the corresponding depolarizing field. In this context, Bi-5517 is akin to so-called hyper-ferroelectrics ${ }^{22}$, whereby soft LO phonons are associated to a large high-frequency dielectric constant and small Born dynamical charges (a feature generally barred by the large gaps and Born charges characteristic of prototypical ferroelectric perovskites). In this sense, Bi-5517 behaves as a limiting case of hyper-ferroelectric, and might thus be considered an instance of self-screened hyper-ferroelectric metal.

In conclusion, we have designed a Bi-based layered-perovskite titanate that presents native metallicity-in the form of a conductive low-dimensional electron gas-and, simultaneously, complies with the requirements of a regular switchable ferroelectric. Besides its conceptual significance, and the fundamental interest of further characterizing the behaviour of $\mathrm{Bi}-5517$ and related materials, our finding opens interesting perspectives for innovative applications. Intriguing possibilities range from the fields of photovoltaics (as a metal, Bi-5517 can be expected to be a good absorber that, simultaneously, features a built-in driving force to separate electrons and holes) to electronics (Bi-5517 may be expected to behave as a heavily $\mathrm{n}$-doped semiconductor strongly responsive to applied fields) or spintronics (there are obvious strategies to construct a spin-polarized, multiferroic version of our ferroelectric metal). We thus hope our work will motivate further investigations of this compound and related ones based on similar strategies to achieve the co-existence of ferroelectricity and metallicity.

\section{Methods}

Simulation details. Our calculations are performed at the first-principles level within the local density approximation ${ }^{23,24}$ to density-functional theory and the projector-augmented wave scheme $\mathrm{e}^{25}$ to treat the interaction between ionic cores and valence electrons, as implemented in the first-principles package VASP ${ }^{26-29}$. The following electrons are explicitly considered in the simulations: Ti's $3 s, 3 p$, $3 d$ and $4 s$; La's $5 s, 5 p, 5 d$ and $6 s$; Bi's $5 d, 6 s$ and $6 p$; O's $2 s$ and $2 p$. The electronic wave functions are represented in plane-wave basis truncated at $500 \mathrm{eV}$. For all self-consistency and force calculations, Brillouin zone integrals are computed on the k-point $6 \times 1 \times 5$ grid, reflecting the elongated shape of the cells ( $a \approx 3.9 \AA$, $b \approx 31.0-55 \AA$ and $c \approx 5.4 \AA$; details are given in Supplementary Table 1.)

For the analysis of the electronic structure, we also use the variational pseudo-self-interaction-corrected density-functional approach ${ }^{30,31}$, which uses ultra-soft pseudopotentials ${ }^{32}$ with plane-wave cutoff of $476 \mathrm{eV}$. DOS calculations use $12 \times 4 \times 8$ grids for Brillouin zone integration.

For transport, we use two approaches: first, the Bloch-Boltzmann transport theory ${ }^{33}$ as implemented in the BoltzTraP code ${ }^{34}$, interpolating the band structure over a $30 \times 14 \times 22$ ab initio calculated k-point values; second, an effective-mass band model that allows an easy inclusion of localized states below the mobility edge. In both cases, the relaxation time needed by Bloch-Boltzmann is calculated by analytic modelling, including the most important scattering contributions (that is, electron-phonon and impurity scattering). The model was previously applied to describe several low-dimensional systems involving titanates, with satisfactory results ${ }^{35-38}$.

Polarization of metals with confined conduction charges. To compute the electronic polarization contribution from the conduction electrons, we use a modified Berry phase ${ }^{16,17,39}$ approach. In its standard version, this approach shows that polarization in crystals is the integrated current flowing through the system as 
atoms displace from the CS $(\lambda=0)$ to the non-CS phase $(\lambda=1)$ :

$$
\Delta P=-\frac{2 e}{8 \pi^{3}} \int_{A_{k}} d k_{\perp}\left(\phi^{(1)}\left(k_{\perp}\right)-\phi^{(0)}\left(k_{\perp}\right)\right)
$$

where $k_{\perp}$ spans the Brillouin zone section, of area $A_{k}$, orthogonal to the polarization direction, and $\phi$ is the Berry phase of the Bloch wavefunctions:

$$
\phi^{(\lambda)}\left(k_{\perp}\right)=\lim _{\mathrm{N} \rightarrow \infty} \mathfrak{T}\left\{\ln \prod_{j=0}^{N-1} \operatorname{det} M_{j, v}^{(\lambda)}\right\}
$$

with

$$
M_{j, v}^{(\lambda)}=\left\langle u_{n k_{j}}^{(\lambda)} \mid u_{n k_{j+1}}^{(\lambda)}\right\rangle
$$

where the $u_{n k_{j}}$ 's are periodic parts of the Bloch wavefunctions, $v$ the number of bands, $n=[1, v]$ and $m=[1, v]$ band indexes, and $k_{j}$ runs over a string of $N$ discrete points from $\Gamma$ to $G_{\|}$, that is the shortest $G$-vector in the direction parallel to the polarization.

In general, the above expressions cannot be applied to a metal, as the Berry phase is well defined (that is, gauge-dependent by unitary rotation) as long as the bands that contribute to the matrix in the above equation are an isolated subgroup (typically the whole valence band manifold of an insulator). Clearly, for a metal, this condition does not hold, as $v$ depends on $k$, that is, the number of occupied bands change with $k$. However, if the system has flat bands along a specific direction (this applies to $\mathrm{Bi}-5517$ since electrons are quite localized within each 5 -unit block along the $\boldsymbol{b}$ axis), then the number of occupied bands only changes with $k_{\perp}$, but not along the string, that is, no band crosses the Fermi level along the $\mathrm{k}$-space string parallel to the insulating direction. It follows that $\phi^{(\lambda)}\left(k_{\perp}\right)$ is well defined for any $k_{\perp}$, while $v$ can change with $k_{\perp}$ with the constraint

$$
Q=\frac{2 e}{A_{k}} \int_{A_{k}} d k_{\perp} v\left(k_{\perp}\right)
$$

on the total electron charge $Q$. In practice $\phi^{(\lambda)}\left(k_{\perp}\right)$ may fluctuate widely with $k_{\perp}$ due to the change in the number of bands contributing to the string, and in turn the $2 \mathrm{D}$ average in the above equation may be slowly convergent. The considerable computing effort required to calculate the Berry phase in large-size systems (such as Bi-5517) suggests adopting a strategy to minimize the contribution of the variable-band-number part. In the specific case of $\mathrm{Bi}-5517$, the latter contribution is limited to a few conduction bands, well separated by a large band gap from the valence bands. We therefore first calculate separately the polarization due to valence bands, which typically converges rapidly over a limited set of $k_{\perp}$ points. For the conduction bands, the polarization is then calculated as the $2 \mathrm{D}$ average of the renormalized phase

$$
\bar{\phi}^{(\lambda)}\left(k_{\perp}\right)=\frac{Q_{\text {cond }}}{2 e v_{\text {cond }}\left(k_{\perp}\right)} \phi^{(\lambda)}\left(k_{\perp}\right)
$$

where $Q_{\text {cond }}$ is the conduction charge and $v_{\text {cond }}$ the number of occupied conduction bands at $k_{\perp}$. With this choice, each $k$-string contribution is renormalized to the same number of electrons, and fluctuates much less with $k_{\perp}$, reducing the effort needed to converge the calculation. Further, in this approximation, even partial band occupancies — as it would correspond to $k_{\perp}$ points at the FS—can be handled by allowing fractional $v_{\text {cond }}\left(k_{\perp}\right)$ values.

Filters. To analyse the potential and charge density in the SLs, we apply well-known averaging processes, described, for example, in refs 7,40 . The filter average features prominently in Fig. 4 of the paper, and is defined as

$$
\overline{\bar{n}}(z)=\frac{1}{L} \int_{z-L / 2}^{z+L / 2} d z^{\prime} \int_{A} n\left(x, y, z^{\prime}\right) d x d y \equiv \frac{1}{L} \int_{z-L / 2}^{z+L / 2} d z^{\prime} \bar{n}\left(z^{\prime}\right)
$$

with $n$ a function (for example, the charge density). It is a one-dimensional squarewave filter of the planar average (implicitly defined by the second equality, and calculated over a sectional area $A$ ) over a window of fixed width $L$, which is its defining parameter. If $L$ is the microscopic periodicity length (assuming such periodicity exists), all microscopic oscillations in the planar average are eliminated. This basically amounts to filtering away all the Fourier components corresponding to microscopic oscillations.

However, the typical potential or charge density in our system has a rather wide spectrum in wavevector space, and filtering all components would entail a complete loss of information. So, in practice, we choose filters such that microscopic oscillations are reduced significantly after a couple of passes at most. We find that applying the filter twice, with $L=d$ and $2 d$, or with $L=3 d$ and $2 d$, gives the best results in terms of oscillation removal, $d$ being the average interplanar distance.

\section{References}

1. Anderson, P. W. \& Blount, E. I. Symmetry considerations on Martensitic Transformations: "Ferroelectric" Metals? Phys. Rev. Lett. 14, 217-219 (1965).

2. Sergienko, I. A. et al. Metallic "Ferroelectricity" in the pyrochlore $\mathrm{Cd}_{2} \mathrm{Re}_{2} \mathrm{O}_{7}$. Phys. Rev. Lett. 92, 065501 (2004).
3. Shi, Y. et al. A Ferroelectric-like structural transition in a metal. Nat. Mater. 12, 1024-1027 (2013).

4. Puggioni, D. \& Rondinelli, J. M. Designing a robustly metallic noncentrosymmetric ruthenate oxide with large thermopower anisotropy. Nat Commun 5, 3432 (2014).

5. Wang, Y. et al. Ferroelectric instability under screened coulomb interactions. Phys. Rev. Lett. 109, 247601 (2012).

6. Junquera, J. \& Ghosez, P. h. Critical thickness for ferroelectricity in perovskite ultrathin films. Nature 422, 506-509 (2003).

7. Ricci, F., Filippetti, A. \& Fiorentini, F. Giant electroresistance and tunable magnetoelectricity in a multiferroic junction. Phys. Rev. B 88, 235416 (2013).

8. Lichtenberg, F., Herrnberger, A., Wiedenmann, K. \& Mannhart, J. Synthesis of perovskite-related layered $\mathrm{A}_{\mathrm{n}} \mathrm{B}_{\mathrm{n}} \mathrm{O}_{3 \mathrm{n}+2}=\mathrm{ABO}_{\mathrm{x}}$ type niobates and titanates and study of their structural, electric and magnetic properties. Prog. Sol. St. Chem. 29, 1-70 (2001).

9. Lichtenberg, F., Herrnberger, A. \& Wiedenmann, K. Synthesis, structural, magnetic and transport properties of layered perovskite-related titanates, niobates and tantalates of the type $\mathrm{A}_{\mathrm{n}} \mathrm{B}_{\mathrm{n}} \mathrm{O}_{3 \mathrm{n}+2}, \mathrm{~A}^{\prime} \mathrm{A}_{\mathrm{k}-1} \mathrm{~B}_{\mathrm{k}} \mathrm{O}_{3 \mathrm{k}+1}$ and $\mathrm{A}_{\mathrm{m}} \mathrm{B}_{\mathrm{m}-1} \mathrm{O}_{3 \mathrm{~m}}$. Prog. Sol. St. Chem. 36, 253-387 (2008).

10. López-Pérez, J. \& Íñiguez, J. Ab initio study of proper topological ferroelectricity in layered perovskite $\mathrm{La}_{2} \mathrm{Ti}_{2} \mathrm{O}_{7}$. Phys. Rev. B 84, 075121 (2011).

11. Scarrozza, M., Filippetti, A. \& Fiorentini, V. Ferromagnetism and orbital order in a topological ferroelectric. Phys. Rev. Lett. 109, 217202 (2012).

12. Scarrozza, M., Filippetti, A. \& Fiorentini, V. Multiferroiticity in vanadium-doped $\mathrm{La}_{2} \mathrm{Ti}_{2} \mathrm{O}_{7}$ : insights from first principles. Eur. Phys. J. B 86, 128 (2013).

13. Williams, $\mathrm{T}$. et al. On the crystal structures of $\mathrm{La}_{2} \mathrm{Ti}_{2} \mathrm{O}_{7}$ and $\mathrm{La}_{5} \mathrm{Ti}_{5} \mathrm{O}_{17}$ : high resolution electron microscopy. J. Sol. St. Chem. 93, 534-548 (1991).

14. Guennou, M., Viret, M. \& Kreisel, J. Bismuth based perovskites as multiferroics. Comptes Rendus Physique 16, 182-192 (2015).

15. Diéguez, O., González-Vázquez, O. E., Wojdeł, J. C. \& Íñiguez, J. First-principles predictions of low-energy phases of multiferroic $\mathrm{BiFeO}_{3}$. Phys. Rev. B 83, 094105 (2011).

16. King-Smith, R. D. \& Vanderbilt, D. Theory of polarization of crystalline solids. Phys. Rev. B 47, 1651-1654 (1993).

17. Resta, R. Macroscopic polarization in crystalline dielectrics: the geometric phase approach. Rev. Mod. Phys. 66, 899-915 (1994).

18. Ghosez, P., Gonze, X. \& Michenaud, J.-P. Coulomb interaction and ferroelectric instability of $\mathrm{BaTiO}_{3}$. Europhys. Lett. 33, 713-718 (1996).

19. Ravindran, P. et al. Theoretical investigation of magnetoelectric behavior in $\mathrm{BiFeO}_{3}$. Phys. Rev. B 74, 224412 (2006).

20. Seshadri, R. \& Hill, N. A. Visualizing the role of Bi 6 s "lone pairs" in the off-center distortion in ferromagnetic $\mathrm{BiMnO}_{3}$. Chem. Mater. 13, 2892-2899 (2001).

21. He, X. et al. Evolution of the electronic and lattice structure with carrier injection in $\mathrm{BiFeO}_{3}$, Preprint at http://arxiv.org/abs/1511.07128 (2015).

22. Garrity, K. F., Rabe, K. M. \& Vanderbilt, D. Hyperferroelectrics: proper ferroelectrics with persistent polarization. Phys. Rev. Lett. 112, 127601 (2014)

23. Ceperley, D. \& Alder, B. J. Ground state of the electron gas by a stochastic method. Phys. Rev. Lett. 45, 566-569 (1980).

24. Perdew, J. P. \& Zunger, A. Self-interaction correction to density-functional approximations for many-electron systems. Phys. Rev. B 23, 5048-5079 (1981)

25. Blöchl, P. E. Projector augmented-wave method. Phys. Rev. B 50, 17953-17979 (1994).

26. Kresse, G. \& Hafner, J. Ab initio molecular dynamics for liquid metals. Phys. Rev. B 47, 558-561 (1993).

27. Kresse, G. \& Furthmüller, J. Efficiency of $a b$-initio total energy calculations for metals and semiconductors using a plane-wave basis set. Comput. Mater. Sci. 6 , 15-50 (1996)

28. Kresse, G. \& Furthmüller, J. Efficient iterative schemes for $a b$ initio total-energy calculations using a plane-wave basis set. Phys. Rev. B 54, 11169-11186 (1996).

29. Kresse, G. \& Joubert, D. From ultrasoft pseudopotentials to the projector augmented-wave method. Phys. Rev. B 59, 1758-1775 (1999).

30. Filippetti, A. et al. Variational pseudo-self- interaction-corrected density functional approach to the $a b$ initio description of correlated solids and molecules. Phys. Rev. B 84, 195127 (2011).

31. Filippetti, A. \& Fiorentini, V. A practical first-principles band-theory approach to the study of correlated materials. Eur. Phys. J. B 71, 139-183 (2009).

32. Vanderbilt, D. Soft self-consistent pseudopotentials in a generalized eigenvalue formalism. Phys. Rev. B 41, 7892-7895 (1990).

33. Allen, P. B. in Quantum Theory of Real Materials (eds Chelikowsky, J. R. \& Louie, S. G.) 219-250 (Kluwer, 1996).

34. Madsen, G. \& Singh, D. BoltzTraP. A code for calculating band-structure dependent quantities. Comput. Phys. Comm 175, 67-71 (2006).

35. Filippetti, A. et al. Thermopower in oxide heterostructures: The im- portance of being multiple-band conductors. Phys. Rev. B 86, 195301 (2012).

36. Delugas, P. et al. Doping-induced dimensional crossover and thermopower burst in Nb-doped $\mathrm{SrTiO}_{3}$ superlattices. Phys. Rev. B 88, 045310 (2013). 
37. Delugas, P. et al. Large band offset as driving force of two-dimensional electron confinement: The case of $\mathrm{SrTiO}_{3} / \mathrm{SrZrO}_{3}$ interface. Phys. Rev. B 88, 115304 (2013).

38. Pallecchi, I. et al. Giant oscillating thermopower at oxide interfaces. Nat. Commun 6, 6678 (2015).

39. Vanderbilt, D. \& King-Smith, R. D. Electric polarization as a bulk quantity and its relation to surface charge. Phys. Rev. B 48, 4442-4455 (1993).

40. Peressi, M., Binggeli, N. \& Baldereschi, A. Band engineering at interfaces: theory and numerical experiments. J. Phys. D Appl. Phys. 31, 1273-1299 (1998).

\section{Acknowledgements}

Work supported in part by MIUR-PRIN 2010 project Oxide (A.F., V.F., P.D., F.R.) Fondazione Banco di Sardegna (A.F., V.F., P.D.), FNR Luxembourg Grant No. FNR/P12/4853155/Kreisel (J.I.), MINECO-Spain Grant No. MAT2013-40581-P (J.I.), CINECA-ISCRA grants (A.F., V.F., F.R., P.D.), CAR of UniCagliari (V.F.). J.I. ran calculations at the CESGA supercomputing center. A.F., V.F., P.D. and F.R. ran calculations at CINECA.

\section{Author contributions}

A.F. and P.D. led the work to characterize the electric, electronic and transport properties of $\mathrm{Bi}_{5} \mathrm{Ti}_{5} \mathrm{O}_{17}$. V.F. and F.R. led the work to characterize the behaviour of finite layers and slabs, and the effects of doping. J.I. led the work to identify $\mathrm{Bi}_{5} \mathrm{Ti}_{5} \mathrm{O}_{17}$ as a candidate ferroelectric metal.

\section{Additional information}

Supplementary Information accompanies this paper at http://www.nature.com/ naturecommunications

Competing financial interests: The authors declare no competing financial interests.

Reprints and permission information is available online at http://npg.nature.com/ reprintsandpermissions/

How to cite this article: Filippetti, A. et al. Prediction of a native ferroelectric metal. Nat. Commun. 7:11211 doi: 10.1038/ncomms11211 (2016).

(c) (i) This work is licensed under a Creative Commons Attribution 4.0 International License. The images or other third party material in this article are included in the article's Creative Commons license, unless indicated otherwise in the credit line; if the material is not included under the Creative Commons license, users will need to obtain permission from the license holder to reproduce the material. To view a copy of this license, visit http://creativecommons.org/licenses/by/4.0/ 